\title{
Sodium Thiosulfate Ameliorates Renovascular Hypertension-Induced Renal Dysfunction and Injury in Rats
}

\author{
Pei-Li Chou ${ }^{a}$ Yin-Shian Chen ${ }^{\text {a }}$ Shiu-Dong Chung ${ }^{b, c}$ Sam-Chi Lin ${ }^{d}$ \\ Chiang-Ting Chien ${ }^{\mathrm{a}}$
}

aDepartment of Life Science, School of Life Science, College of Science, National Taiwan Normal University, Taipei, Taiwan, ROC; 'bivision of Urology, Department of Surgery, Far-Eastern Memorial Hospital, Banciao, New Taipei City, Taiwan, ROC; 'CGraduate Program in Biomedical Informatics, College of Informatics, Yuan-Ze University, Chung-Li, Taoyuan City, Taiwan, ROC; ${ }^{d}$ Division of Renal Section, Department of Internal Medicine, Taipei Hospital, Ministry of Health and Welfare, New Taipei City, Taiwan, ROC

\section{Keywords}

Sodium thiosulfate $\cdot$ Renovascular hypertension $\cdot$ Reactive oxygen species · Antioxidants · Apoptosis

\begin{abstract}
Background/Aims: Arterial stenosis activates the renin-angiotensin-aldosterone system subsequently resulting in renovascular hypertension (RVHT) and renal oxidative injury. We explored the effect of sodium thiosulfate (STS, $\mathrm{Na}_{2} \mathrm{~S}_{2} \mathrm{O}_{3}$ ), a developed antioxidant in clinical trial, on RVHT-induced hypertension and renal oxidative injury in rats. Methods: We induced RVHT in male Wistar rats with bilaterally partial ligation of renal arteries in the 2-kidney 2-clip model. We evaluated the STS effect on RVHT-induced oxidative injury and apoptosis by a chemiluminescence amplification method, Western blot, and immunohistochemistry. Results: We found STS displayed a dose-dependent antioxidant $\mathrm{H}_{2} \mathrm{O}_{2}$ activity and adapted the maximal scavenging $\mathrm{H}_{2} \mathrm{O}_{2}$ activity of STS at the dosage of $0.1 \mathrm{~g} / \mathrm{kg}$ intraperitoneally 3 times/week for 4 weeks in RVHT rats. RVHT induced a significant elevation of arterial blood pressure, blood reactive oxygen species amount, neutrophil infiltration, 4-HNE and NADPH oxidase gp91 expression, Bax/Bcl-2/poly(ADP-ribose) polymerase
\end{abstract}

$\begin{array}{ll}\text { karger@karger.com } & \text { (C) } 2020 \text { The Author(s). } \\ \text { www.karger.com/kbr } & \text { Published by S. Karger AG, Basel } \\ & \text { This article is licensed under the Creative Commons Attribution- } \\ \text { Karger } & \begin{array}{l}\text { NonCommercial-NoDerivatives 4.0 International License (CC BY- } \\ \text { NC-ND) (http://www.karger.com/Services/OpenAccessLicense). }\end{array} \\ \text { Usage and distribution for commercial purposes as well as any dis- } \\ \text { tribution of modified material requires written permission. }\end{array}$

(PARP)-mediated apoptosis formation, blue Masson-stained fibrosis, and urinary protein level. STS treatment significantly reduced hypertension, oxidative stress, neutrophil infiltration, fibrosis, and Bax/Bcl-2/PARP-mediated apoptosis formation and depressed the urinary protein level in the RVHT models. Conclusion: Our results suggest that STS treatment could ameliorate RVHT hypertension and renal oxidative injury through antioxidant, antifibrotic, and antiapoptotic mechanisms. (c) 2020 The Author(s) Published by S. Karger AG, Basel

\section{Introduction}

Hypertension is a major health problem associated with structural and functional modifications of the vasculature. Renovascular hypertension (RVHT) is one type of secondary hypertension due to the renal arterial stenosis-induced renin-angiotensin-aldosterone system (RAAS) activation, sodium/volume overload, and sympathetic nervous system excitation [1-3]. The activation

Chiang-Ting Chien

Department of Life Science, School of Life Science

College of Science, National Taiwan Normal University

No. 88, Sect 4 of Tingzhou Rd., Tapei City 11677, Taiwan (ROC)

ctchien@ntnu.edu.tw 
of RAAS is closely related to the progression and development of cardiovascular disease and chronic kidney disease [4]. Increased angiotensin II not only increases blood pressure but also produces reactive oxygen species (ROS) via the activation of NADPH oxidase [5]. Excessive ROS promotes the vasoconstriction and proliferation of vascular smooth muscle cells and induces endothelial cell dysfunction and inflammatory response in the vessel wall, which can cause heart or kidney dysfunction and injury [6]. On the other hand, exacerbated ROS generation from the mitochondria or other intracellular compartments may induce one type of programmed cell death, apoptosis, via the execution by Bax/Bcl-2/caspase/poly(ADP-ribose) polymerase (PARP) signaling leading to renal oxidative injury [7].

In translational medicine, the discovery of new drugs or new potential uses for currently available drugs is crucial for treating the resistant hypertension associated with renal artery stenosis. Sodium thiosulfate (STS), a major metabolite of $\mathrm{H}_{2} \mathrm{~S}$, has been proven safe [8] for the treatment of calciphylaxis [9]. In addition, STS is suggested to be an antioxidant [9] and useful in case of cyanide poisoning [10] or cisplatin toxicity [11]. In clinical trials, vasodilating properties of STS itself have been reported in alleviating cerebral vasospasm [12] and hypertension and renal injury [13]. Moreover, direct evidence implicated that STS attenuates angiotensin II-induced hypertension, proteinuria, and renal damage [13] and ameliorates oxidative stress and preserves renal function in hyperoxaluric rats [14]. Oxidative stress plays a pathophysiologic role in hypertension and renal arterial stenosis. We therefore suggest that antioxidant and vasodilating STS could confer therapeutic potential in improving RVHT-induced hypertension and renal injury.

Several methods to induce hypertension with renal arterial constriction included 1-kidney 1-clip (1K1C), 2-kidney 1-clip (2K1C), and 2-kidney 2-clip (2K2C) methods [15]. 1K1C RVHT had a high mortality because of malignant hypertension, the $2 \mathrm{~K} 1 \mathrm{C}$ RVHT model was hard to induce hypertension, and the $2 \mathrm{~K} 2 \mathrm{C}$ RVHT model easily achieved hypertension with very few deaths as a result of acute renal failure [15]. Therefore, in this study, we employed the 2K2C RVHT rat model to explore STS's protective effects and mechanisms on RVHT-induced systemic hypertension, kidney dysfunction, and oxidative damage. Moreover, we delineated the relationship between the beneficial role of STS and the antioxidant and antiapoptotic pathways in the hypertensive progression.

\section{Methods and Materials}

\section{Animals}

Male Wistar rats (200-250 g) were purchased from BioLASCO Taiwan Co. Ltd. (I-Lan, Taiwan) and housed at the Experimental Animal Center, National Taiwan Normal University, at a constant temperature and with a consistent light cycle (light from 07:00 to 18:00 h). The rats were fed standard chow (Laboratory Rodent Diet 5001 containing $0.4 \%$ sodium) and tap water ad libitum. All surgical and experimental procedures were approved by the Institutional Animal Care and Use Committee of National Taiwan Normal University (Approval No. 107026, September 26, 2018) and were in accordance with the guidelines of the National Science Council of Republic of China.

\section{RVHT Induction Model}

We adapted bilateral renal arterial partial ligation-induced RVHT in this study. Under $2.5 \%$ isoflurane (Panion \& BF Biotech Inc., Taoyuan, Taiwan) anesthesia, RVHT was induced in the bilateral kidneys using a $2 \mathrm{~K} 2 \mathrm{C}$ method, as described by Zeng et al. [15]. In brief, under anesthesia, the median longitudinal incision on the abdominal skin was performed, and then the roots of both right and left renal arteries were constricted by placing ring-shaped silver clips with an inner diameter of $0.30 \mathrm{~mm}$ to induce hypertension. Under surgery of the induction of RVHT, the anesthetized rats were evaluated with the maintenance of deep anesthesia by the persistence of miotic pupils as judged from frequent inspection [16]. Body temperature was maintained at $37^{\circ} \mathrm{C}$ with a heat lamp. We also determined the renal arterial blood flow by placing the renal artery on a blood flow probe and connected to a transonic flow meter (T420; Transonic System Inc., Ithaca, NY, USA) and recorded on a Power Lab system (ML870 PowerLab 8/30; ADInstruments Pty Ltd, NSW, Australia). Renal arterial blood flow was continuously recorded during the baseline period and the clipping period $10 \mathrm{~min}$ for confirmation of the reduction in renal arterial blood flow. Similar surgery was also performed on sham rats including median longitudinal incision on the abdominal skin and isolation of bilateral renal arteries except the placement of silver clips on bilateral renal arteries. After RVHT induction and renal arterial blood flow measurement, all the rats were sutured and recovered.

\section{Hypertension Model and Drug Administration}

After 8-week RVHT induction, these conscious $2 \mathrm{~K} 2 \mathrm{C}$ rats with systolic blood pressure higher than $140 \mathrm{~mm} \mathrm{Hg}$ determined by tail-cuff plethysmography (Blood Pressure Analysis System, BP2000 SERIES II; Vistech Systems, Inc.) and without stroke symptoms were selected for further 4-week STS treatment experiment. We administered STS (Alfa Aesar, Lancashire, UK) at the dosage of $0.1 \mathrm{~g} / \mathrm{kg}$ intraperitoneally 3 times per week for 4 weeks. The sham and RVHT groups were administered intraperitoneal saline. After 4-week STS treatment, under 2.5\% isofluorane (Panion \& BF Biotech Inc., Taoyuan, Taiwan), the left femoral artery of the anesthetized rats was catheterized with a PE-50 catheter connected with a pressure transducer to continuously record arterial blood pressure on the PowerLab system (ML870 PowerLab 8/30; ADInstruments Pty Ltd). After finishing physiologic parameter determination, the arterial blood was collected for renal functional determination. All the rats were then sacrificed with intravenous $\mathrm{KCl}$. The 2 kidneys were resected and divided into 2 parts. One part
Chou et al. 
was stored in $10 \%$ neutral buffered formalin for immunohistochemistry and in situ pathologic assay, and the other was quickly frozen in liquid nitrogen and stored at $-70^{\circ} \mathrm{C}$ for protein isolation.

In vitro Recording for STS on Scavenging ROS Activity

In the part one study, the scavenging ROS level of the different concentrations of STS was measured by the luminol-amplification chemiluminescence detection method as described previously [17]. In brief, $200 \mu \mathrm{L}$ of freshly prepared STS solution (from 0.0001 to $0.2 \mathrm{~g}$ ) was mixed with $0.5 \mathrm{~mL}$ of $0.1 \mathrm{mmol} / \mathrm{L}$ luminol (5-amino-2,3-dihydro-1,4-phthalazinedione; Sigma Chemical Co., St. Louis, MO, USA) and $0.1 \mathrm{~mL}$ of $\mathrm{H}_{2} \mathrm{O}_{2}(0.03 \%)$ and was analyzed with a chemiluminescence analyzing system (CLA-ID3; Tohoku Electronic Inc. Co., Sendai, Japan). The chemiluminescence signals emitted from the mixture of STS solution and luminol, which represented the hydrogen peroxide content in the mixture, were recorded. The enhanced chemiluminescent signals from the sample-luminol- $\mathrm{H}_{2} \mathrm{O}_{2}$ mixture were recorded for $300 \mathrm{~s}$. The total chemiluminescent (CL) counts were calculated by the area under the curve.

In the part II study, 3 groups of rats ( $n=6$ in each group) were used for measurement of ROS in whole-blood samples. The blood samples $(0.2 \mathrm{~mL})$ from the left femoral artery were obtained. The blood samples were wrapped in aluminum foil and kept on ice until CL measurement. Immediately before CL measurement, $0.1 \mathrm{~mL}$ of phosphate-buffered saline ( $\mathrm{pH} 7.4$ ) was added to $0.2 \mathrm{~mL}$ of blood sample. The CL was measured in a completely dark chamber of the chemiluminescence analyzing system (CLA-ID3). After 100 -s background level determination, $0.5 \mathrm{~mL}$ of $0.1 \mathrm{mmol} / \mathrm{L} \mathrm{lu}$ minol in phosphate-buffered saline $(\mathrm{pH} 7.4)$ was injected into the sample. The CL was monitored continuously for an additional 300 s. The total amount of CL was calculated by integrating of the area under the curve and subtracting it from the background level. The assay was performed in duplicate for each sample and was expressed as CL counts/10 s for blood CL.

\section{Western Blotting}

The kidneys were ground to powder in liquid nitrogen, and then the powder was lysed in RIPA buffer (Bio Basic, Amherst, NY, USA) supplemented with protease inhibitor (Roche, Basel, Switzerland) for $10 \mathrm{~min}$ at $4^{\circ} \mathrm{C}$. The detection of signals was done using Western Lightning Plus-ECL (PerkinElmer, Waltham, MA, USA). The expression levels of apoptosis-related proteins including Bcl2, Bax, and PARP and 4-hydroxynonenal (4-HNE) were analyzed by Western blotting in kidney tissues. The Western blotting method has been described elsewhere [7]. Antibodies against 4-HNE (bs-6313R, 1:200; Bioss, Woburn, MA, USA), Bax (bs-0032R, 1:100; Bioss, Mass, USA), Bcl-2 (Transduction; Bluegrass-Lexington, KY, USA), PARP (\#9532, 1:1,000; Cell Signaling Technology, Danvers, MA, USA), and $\beta$-actin (Sigma, St. Louis, MO, USA) were used. The density of the band with the appropriate molecular mass was determined semiquantitatively by densitometry using an image analyzing system (Alpha Innotech, San Leandro, CA, USA).

Secondary antibodies included horseradish peroxidase (HRP)conjugated goat anti-mouse IgG, HRP-conjugated rabbit anti-goat IgG, and HRP-conjugated goat anti-rabbit IgG (all for 1:10,000; all from Southern Biotech Laboratories, Birmingham, AL, USA). For the quantitative comparison of the protein expression levels, intensities of specific bands corresponding to the proteins of interest were measured using densitometry analysis.

Sodium Thiosulfate and Renovascular Hypertension

\section{Histologic Studies}

Tissue samples were fixed in $4 \%$ paraformaldehyde and embedded in paraffin. Then, $4-\mu \mathrm{m}$ sections were obtained and stained with hematoxylin and eosin.

\section{Detection of Oxidative Stress and Apoptosis in Damaged} Kidneys

To examine the effect of STS on RVHT kidneys, several oxidative stress parameters, 4-HNE (ab46545; Abcam, UK), proapoptotic Bax (ab32503; Abcam, UK), antiapoptotic Bcl-2 (3498; Cell Signaling, USA), and PARP (9542; Cell Signaling, USA), and terminal deoxynucleotidyl transferase dUTP nick end labeling (TUNEL assay) were performed to investigate the presence and extent of lipid peroxidation and apoptosis formation in RVHT kidneys. The technique has been reported previously [7]. In brief, renal sections $(4 \mu \mathrm{m})$ were prepared, deparaffinised, and stained with hematoxylin and eosin, Bax, Bcl-2, and PARP stain. A biotinylated secondary antibody (Dako, Botany, NSW, Australia) was then applied followed by streptavidin conjugated to HRP (Dako). The chromogen used was Dako liquid diaminobenzene. Twenty highpower $(\times 400)$ fields were randomly selected for each section, and the value of each oxidative stress was analyzed using Sonix Image Setup (Sonix Technology Co., Ltd., Hinschu, Taiwan, ROC) containing image analyzing software Carl Zeiss AxioVision Rel.4.8.2 (Future Optics Sci. \& Tech. Co., Ltd., Hangzhou, China). The apoptotic index was calculated as the number of TUNEL-positive nuclei per high-power field $(\times 400)$.

\section{Biochemical Parameters in Blood and Urine}

We evaluated the STS effect on urinary protein, BUN, and $\mathrm{Cr}$ in these 3 groups of animals. Urine was directly obtained from the urinary bladder of the anesthetized rats for urinary protein determination by a BCA protein assay kit (Bio-Rad, Hercules, CA, USA). Arterial blood was collected for ROS determination and renal function determination (BUN and $\mathrm{Cr}$ ).

\section{Statistical Analysis}

All values were expressed as mean \pm standard error mean. Data were subjected to 1-way analysis of variance, followed by Duncan's multiple-range test for assessment of the difference among groups. Differences within groups were evaluated by the paired $t$ test. Differences were regarded as significant if $p<0.05$ was attained. Statistical analyses were performed using SPSS 18.0 statistics software (IBM Corp., Armonk, NY, USA).

\section{Results}

\section{STS Dose-Dependently Decreased $\mathrm{H}_{2} \mathrm{O}_{2}$ Amount and} Inhibited NADPH Oxidase gp91

As shown in Figure 1A, STS dose-dependently and significantly reduced $\mathrm{H}_{2} \mathrm{O}_{2}$ amount. Our data further displayed that STS displays a maximal scavenging $\mathrm{H}_{2} \mathrm{O}_{2}$ effect at the dosage of $0.1 \mathrm{~g}$, which is a safe dose $<0.25 \mathrm{~g} / \mathrm{kg}$ for human clinical trial. Therefore, we used the dosage of $0.1 \mathrm{~g} / \mathrm{kg}$ of STS in this study. We explored the STS effect on renal NADPH oxidase gp91, a superoxide anion-pro- 
Fig. 1. A The effect of STS on $\mathrm{H}_{2} \mathrm{O}_{2}$ scavenging activity. Data show that STS displays a dose-dependent manner in scavenging $\mathrm{H}_{2} \mathrm{O}_{2}$ activity, and a maximal effect is at the dosage of $0.1 \mathrm{~g} .{ }^{*} p<0.05$ versus the saline control; A, $p<0.05$ versus $0.0001-\mathrm{g}$ STS; $\mathbf{B}, p<0.05$ versus 0.01 -g STS. B The effect of STS on renal NADPH oxidase gp91 expression in the sham, RVHT, and RVHT STS groups. RVHT enhanced renal gp91 expression, whereas STS inhibited RVHT-enhanced gp91 expression. Data are expressed as mean $\pm \operatorname{SEM}(n=3) .{ }^{\star} p<$ 0.05 versus the sham group, ${ }^{\#} p<0.05$ versus the RVHT group. STS, sodium thiosulfate; RVHT, renovascular hypertension; $\mathrm{CL}$, chemiluminescent.

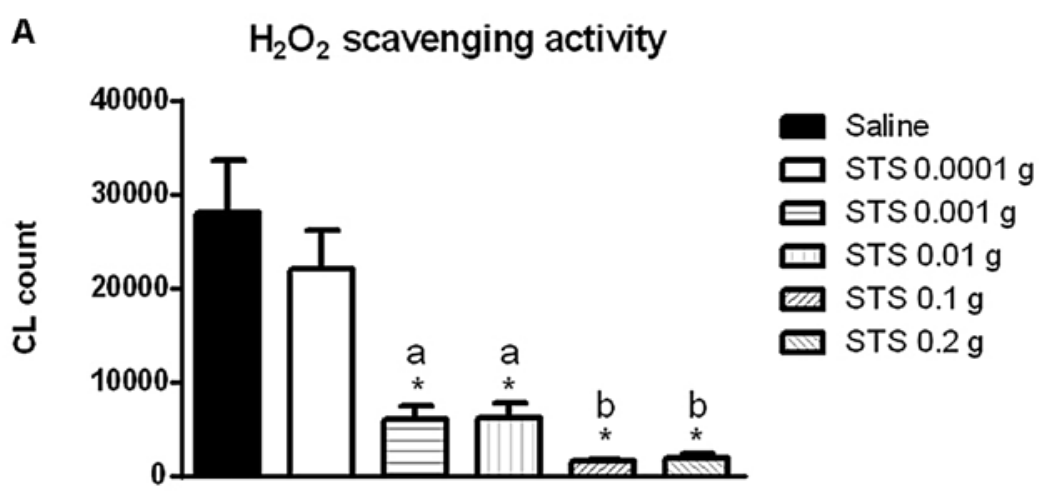

B
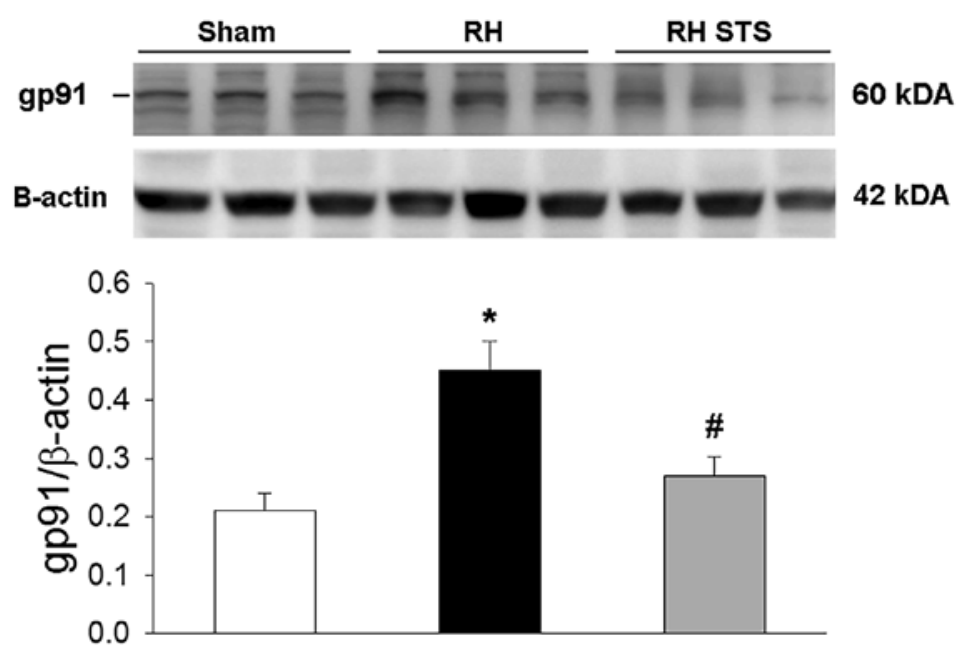

ducing enzyme, expression in the sham, RVHT, and RVHT STS groups. Our results showed that RVHT significantly enhanced NADPH oxidase gp91 in the RVHT and RVHT STS kidneys (Fig. 1B). However, STS treatment significantly reduced gp91 expression in the RVHT STS kidneys versus that in the RVHT kidneys.

\section{RVHT Model}

As shown in Figure 2A and B, we determined the right and left renal arterial blood flow in response to bilateral renal arterial partial ligation in the anesthetized rats with a renal arterial flow probe. The left or right renal arterial blood flow was significantly decreased to a half value of the renal blood flow after partial ligation. After 8 weeks of the $2 \mathrm{~K} 2 \mathrm{C}$ model, the systemic arterial blood pressure was significantly increased to $220 \mathrm{~mm} \mathrm{Hg}$ in the RVHT rats as compared to the $120 \mathrm{~mm} \mathrm{Hg}$ of arterial blood pressure in the control rats (Fig. 2C). The level of arte- rial blood pressure in RVHT rats was $188.9 \%$ of the sham controls (the change was increased by $89.9 \%$ from the sham original) and that in RVHT STS rats was $171.2 \%$ of the sham controls (the change was increased by $71.2 \%$ from the sham original). In comparison with the arterial blood pressure of the sham controls, the difference of increased arterial blood pressure level between RVHT and RVHT STS rats calculated in percent points was $17.7 \%$. A decreased arterial blood pressure degree by STS treatment is not very much.

\section{STS Effect on RVHT-Induced Renal Dysfunction}

We explored the STS effect on RVHT-induced renal dysfunction including urinary protein, $\mathrm{BUN}$, and $\mathrm{Cr}$ in this study. As shown in Figure 3A, the level of urinary protein was only significantly increased by $79.7 \%$ in RVHT rats versus sham controls. The urinary protein level was not significantly increased between STS-treated
44
Chou et al. 
Fig. 2. Evaluation of the $2 \mathrm{~K} 2 \mathrm{C}$ model on right or left renal blood flow for developing RVHT. Partial ligation of the renal artery led to a near $50 \%$ reduction of left $(\mathbf{A})$ and right (B) renal blood flow measured by a flow probe. The $2 \mathrm{~K} 2 \mathrm{C}$ model induced hypertension by elevated $88.9 \%$ of arterial blood pressure in RVHT and $71.2 \%$ in the RVHT STS group versus the sham group (C), whereas STS treatment significantly depressed $9.3 \%$ of arterial blood pressure in the RVHT STS group versus the RVHT group. Data are expressed as mean \pm SEM $(n=6) .{ }^{*} p<0.05$ versus the sham group, ${ }^{\#} p<0.05$ versus the RVHT group. $2 \mathrm{~K} 2 \mathrm{C}$, 2-kidney 2-clip; RVHT, renovascular hypertension; STS, sodium thiosulfate.
A
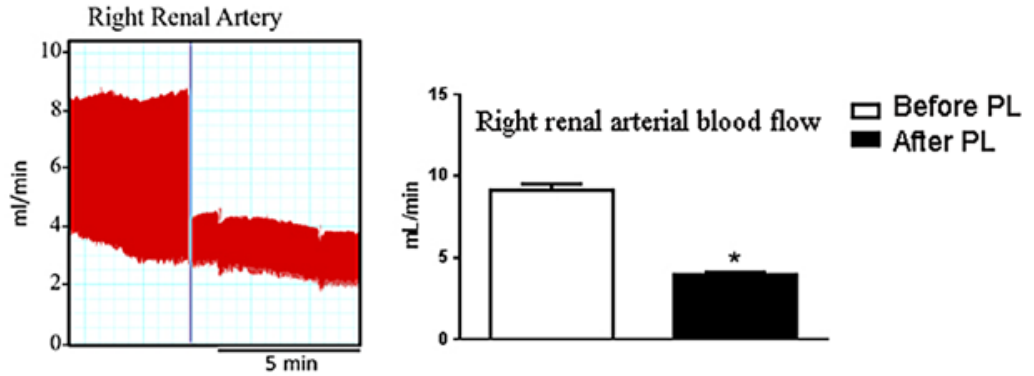

B
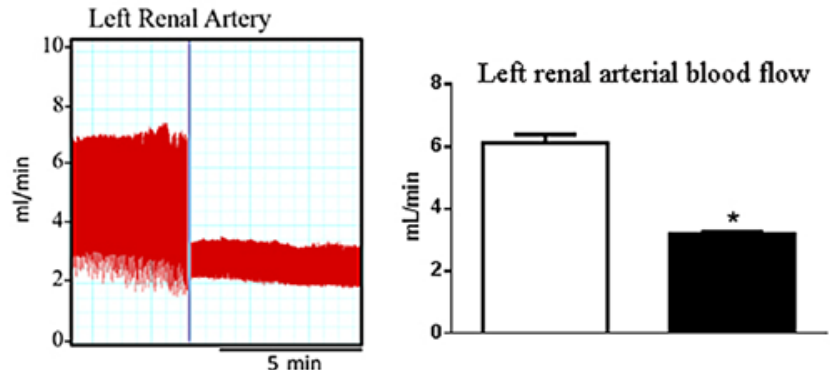

口 Before PL

- After PL

$\mathrm{C}$

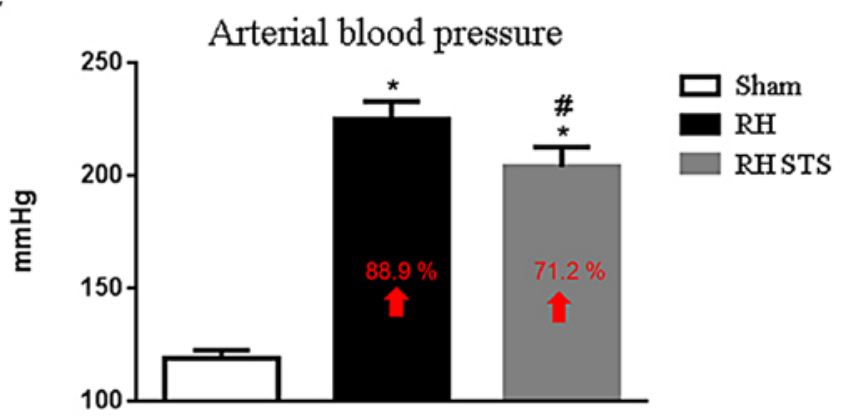

RVHT rats and sham controls. However, STS significantly depressed the elevated urinary protein level by $36.9 \%$ as compared to RVHT rats. Our RVHT model did not significantly affect the level of BUN (Fig. 3C) and $\mathrm{Cr}$ (Fig. 3D), and STS had no effect on the BUN and Cr levels in RVHT rats.

\section{Blood ROS Amount in RVHT Rats}

We used the ultrasensitive chemiluminescence amplification technique to determine the blood ROS amount in these animals. As shown in Figure 3B, the blood ROS amount was significantly $(p<0.05)$ increased in the RVHT rats $(9,990 \pm 517$ counts/10 s) as compared to the sham rats $(2,809 \pm 132$ counts/10 s). STS-treated RVHT rats displayed a significantly depressed blood ROS amount $(4,959 \pm 326$ counts/10 s) as compared to that of
RVHT rats $(9,990 \pm 517$ counts/10 s). The blood ROS level was increased by $255 \%$ in RVHT rats, and STS efficiently depressed the increased blood ROS to $50.4 \%$ in the RVHT STS rats.

\section{Effect of STS on Leukocyte Infiltration in the RVHT}

\section{Kidneys by H\&E Stain}

We examined the leukocyte infiltration in the cortex and medulla of these 3 groups of rats. In the sham rats, there were few leukocyte infiltrations in the cortex and medulla (Fig. 4A). In the RVHT kidneys, there were markedly increased leukocytes infiltrated in the cortex and medulla. In the STS-treated RVHT kidneys, the infiltrated leukocytes were significantly $(p<0.05)$ reduced as compared to RVHT kidneys (Fig. 4C). 


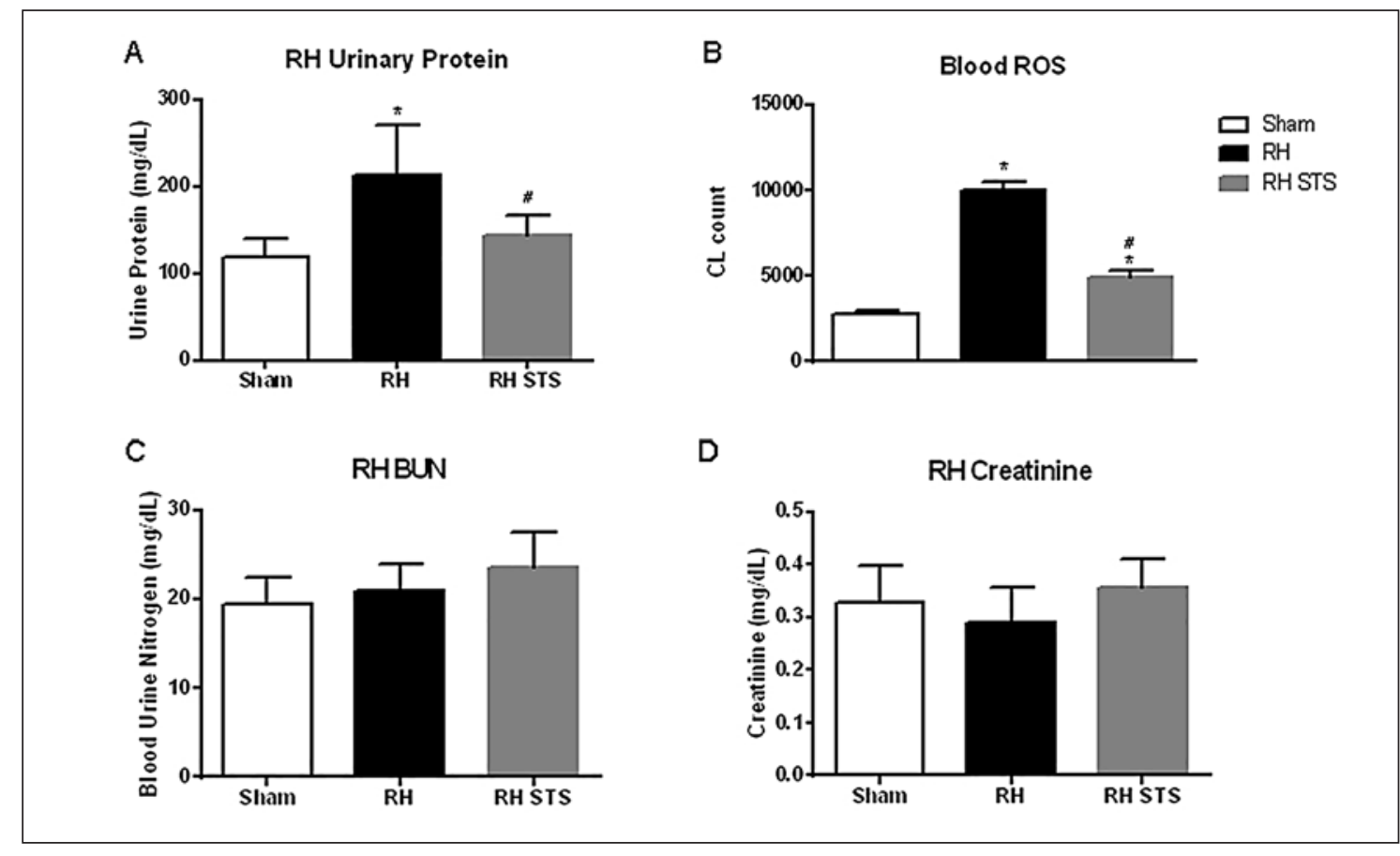

Fig. 3. The STS effect on RVHT-induced renal dysfunction and blood ROS amount. The effect of STS on urinary protein $(\mathbf{A})$, blood ROS amount $(\mathbf{B}), \mathrm{BUN}(\mathbf{C})$, and $\mathrm{Cr}(\mathbf{D})$ was denoted in the RVHT rats. Increased urinary protein and blood ROS amount were found in the RVHT rats, whereas the increased level of urinary protein and blood ROS amount was attenuated in STS-treated RVHT rats (RVHT STS). Data are expressed as mean \pm SEM $(n=6) .{ }^{*} p<0.05$ versus sham. ${ }^{*} p<0.05$ versus RVHT. STS, sodium thiosulfate; RVHT, renovascular hypertension; ROS, reactive oxygen species; $\mathrm{CL}$, chemiluminescent.

Effect of STS on Renal Fibrosis in the RVHT Kidneys by Masson Stain

To explore the STS effect on renal fibrosis in the RVHT kidneys, we used Masson stain to determine the blue collagen deposits in these rats. As shown in Figure 4B, blue Masson stain was markedly found in the cortex and medulla of the RVHT kidneys as compared to sham kidneys. STS treatment significantly $(p<0.05)$ reduced blue Masson stain in the cortex and medulla versus RVHT kidneys (Fig. 4D).

\section{Effect of STS on Renal 4-HNE Expression in RVHT}

Kidneys by Western Blot and Immunohistochemistry

We determined the STS effect on renal 4-HNE expression in RVHT kidneys by Western blot and immunohistochemistry. Our data showed that RVHT injury significantly enhanced renal 4 -HNE (increased by $20.1 \%$ ) expression, a lipid peroxidation biomarker, as compared to that in sham kidneys (Fig. 5A). STS treatment significantly depressed renal 4-HNE expression by $12.8 \%$ in RVHT with the STS group versus the RVHT group. The data from immunohistochemistry also displayed an increase in brown 4-HNE expression in the cortex including glomeruli and tubules and in the medulla of renal distal tubules in the RVHT kidneys as compared to sham kidneys (Fig. 5B). STS treatment efficiently reduced brown 4-HNE expression in both the cortex and medulla (renal tubules and glomeruli) in the RVHT with STS kidneys versus RVHT kidneys.

\section{Effect of STS on Renal Apoptosis-Related Mechanism in RVHT Kidneys by Western Blot and} Immunohistochemistry

We determined the effect of STS on RVHT-evoked apoptosis formation in the kidneys by Western blot and immunohistochemistry. Our data showed that the renal Bax expression was significantly $(p<0.05)$ increased in RVHT kidneys as compared to sham kidneys (Fig. 6A). STS treatment significantly reduced the Bax expression between the RVHT and RVHT STS groups. However, the renal Bcl-2 expression was not significantly different among the sham, RVHT, and RVHT STS groups. We used
Chou et al. 


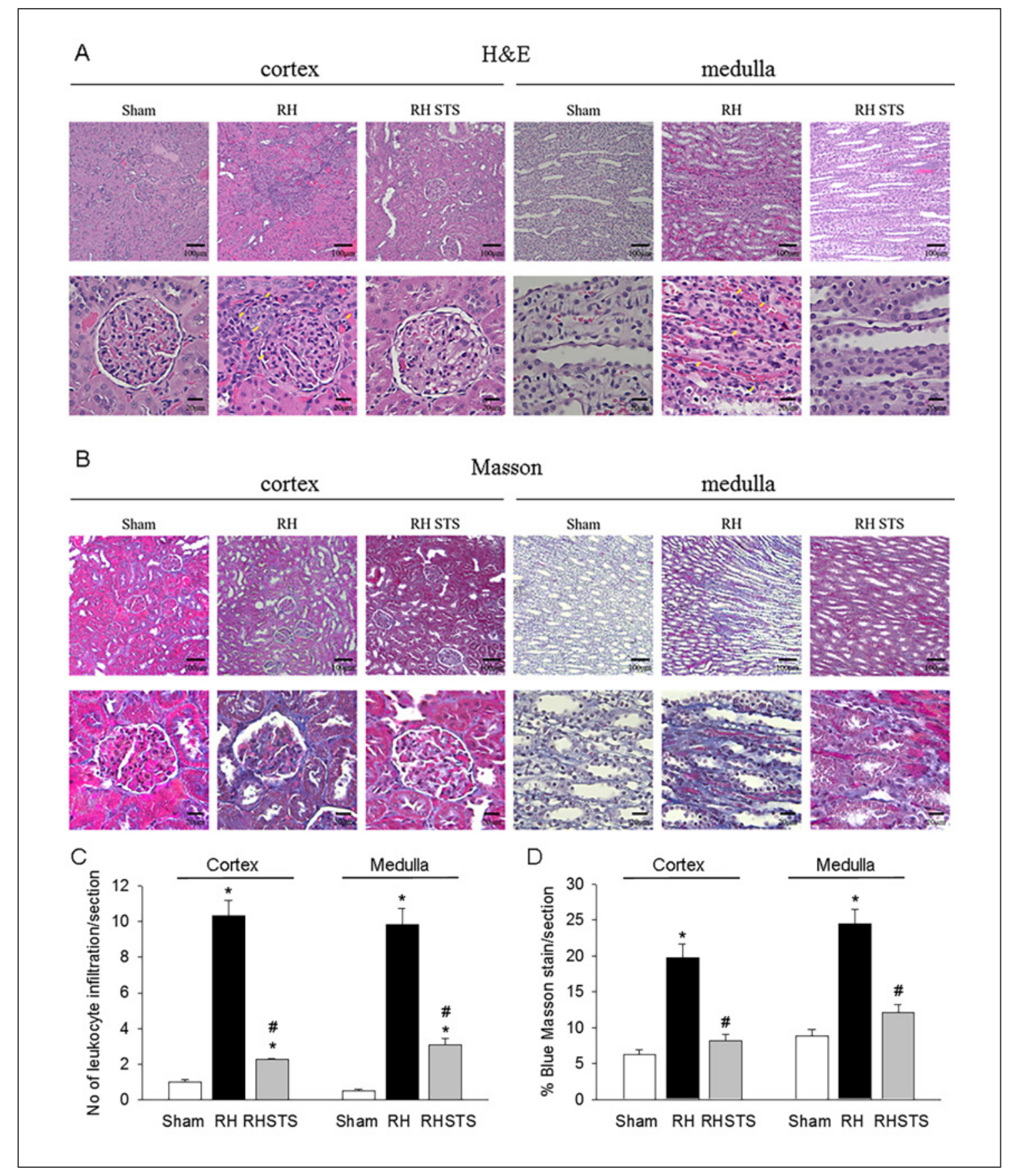

Fig. 4. Histologic graphs from $H \& E$ stain (A) and Masson stain (B) were displayed in sham, RVHT, and STStreated RVHT (RVHT STS) rats. A The increased leukocyte infiltration (yellow arrows) is indicated in the cortex and medulla of the RVHT kidney. B Increased Masson stain by blue color is indicated in the cortex and medulla of the RVHT kidney. The mean data of the number of leukocyte infiltration and \% of blue Masson stain for each section are indicated in $\mathbf{C}$, $\mathbf{D}$, respectively. Data are expressed as mean $\pm \operatorname{SEM}(n=6) .{ }^{\star} p<0.05$ versus sham. ${ }^{\#} p<0.05$ versus RVHT. STS, sodium thiosulfate; RVHT, renovascular hypertension.

Sodium Thiosulfate and Renovascular Hypertension
Kidney Blood Press Res 2021;46:41-52 DOI: 10.1159/000510047 


\section{A}

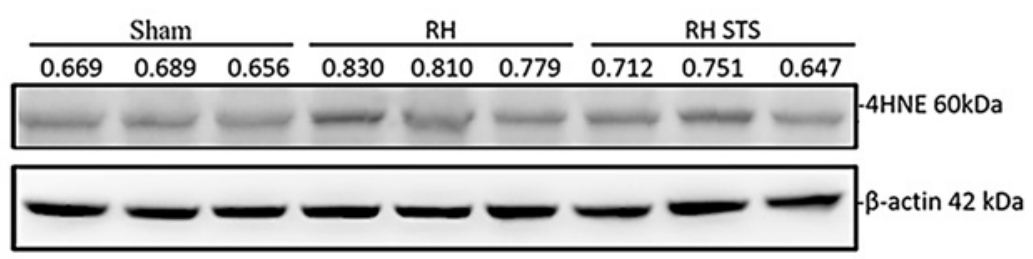

RH-4HNE

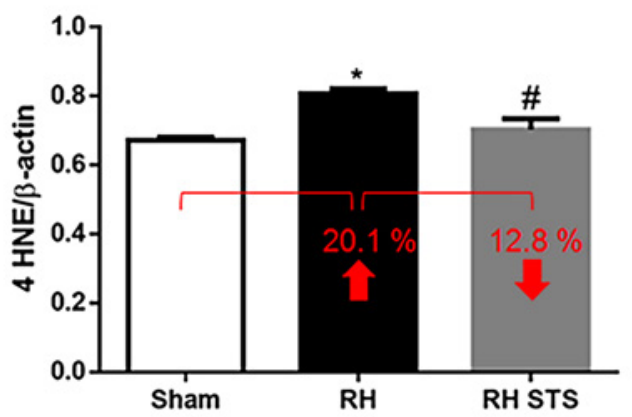

B

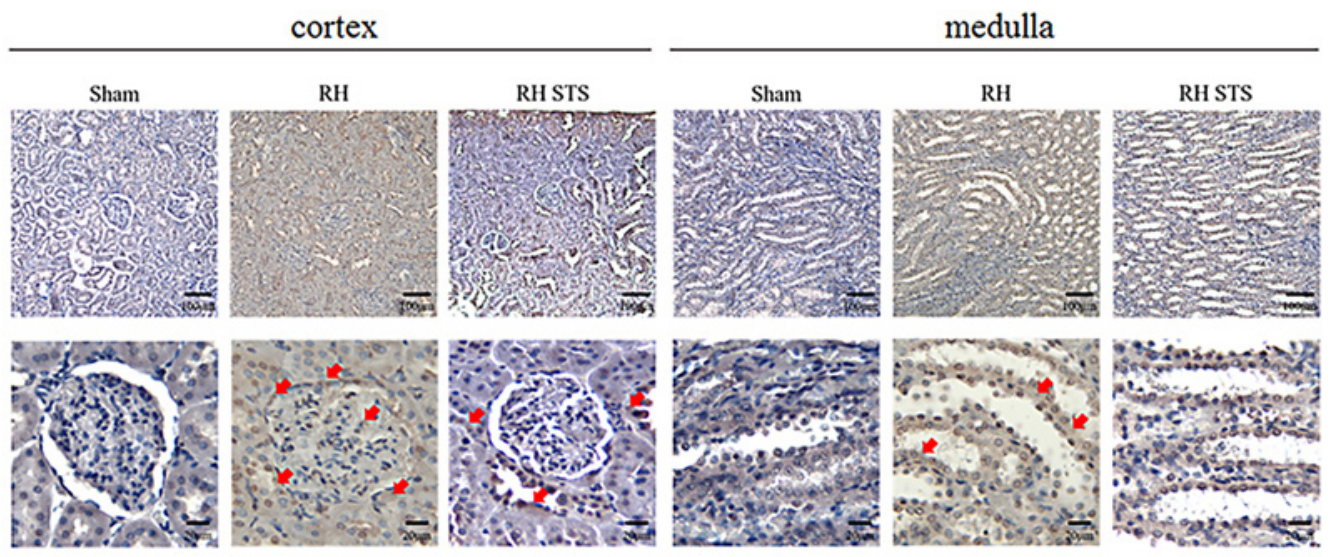

Fig. 5. A Western blot of 4-HNE expression in RVHT-induced kidney injury. B Immunohistochemistry of $4-\mathrm{HNE}$ is indicated in the cortex and medulla of the sham, RVHT, and RVHT STS kidneys. Data are expressed as mean \pm SEM $(n=3) .{ }^{*} p<0.05$ versus sham. ${ }^{*} p<0.05$ versus RVHT. STS, sodium thiosulfate; RVHT, renovascular hypertension.

the $\mathrm{Bax} / \mathrm{Bcl}-2$ ratio to examine the possibly apoptotic mechanisms in the damaged kidneys. Our data found that the ratio of $\mathrm{Bax} / \mathrm{Bcl}-2$ was increased by $49.9 \%$ in the RVHT group versus the sham group. STS treatment significantly attenuated the increased $\mathrm{Bax} / \mathrm{Bcl}-2$ ratio by $30 \%$ in the RVHT STS group versus the RVHT group. The renal Bax immunohistochemistry (Fig. 6B) also displayed a consistent finding with the Western blot data.

We further examined the effect of STS on RVHTevoked PARP expression by Western blot and TUNEL immunohistochemistry. Our data showed that the renal PARP expression was significantly $(p<0.05)$ increased by $28.8 \%$ in the RVHT group as compared to the sham group (Fig. 7A). STS also significantly depressed PARP expression by $16.2 \%$ in RVHT STS kidneys as compared to RVHT kidneys. We further examined the renal TUNEL immunohistochemistry in the cortex (Fig. 7B) and medulla (Fig. 7C). These data showed a significant increase in TUNEL-positive stain cells in the cortex $(1,028.6 \%$ increase in the RVHT group vs. the sham 
A
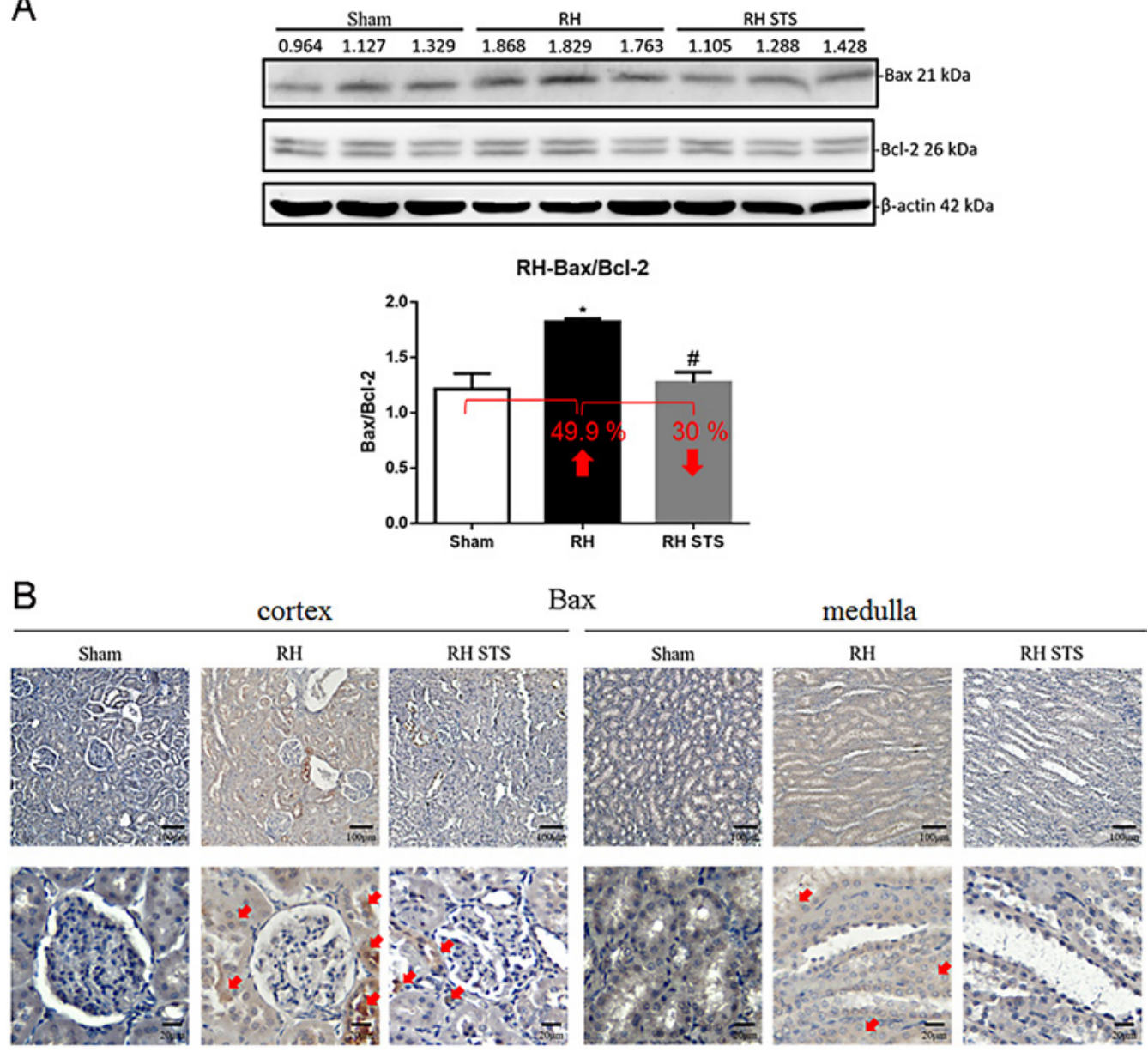

Fig. 6. A Western blot of Bax and Bcl-2 expression in RVHT-induced kidney injury. B Immunohistochemistry of Bax is indicated in the cortex and medulla of the sham, RVHT, and RVHT STS kidneys. Data are expressed as mean \pm SEM $(n=3) .{ }^{\star} p<0.05$ versus sham. ${ }^{*} p<0.05$ versus RVHT. STS, sodium thiosulfate; RVHT, renovascular hypertension.

group) and medulla $(1,560 \%$ increase in the RVHT group vs. the sham group), respectively. RVHT STS kidneys also demonstrated a significant increase in TUNEL apoptosis-positive stain cells in the cortex (514.3\% increase in the RVHT STS group vs. the sham group) and medulla (720\% increase in the RVHT STS group vs. the sham group). However, as compared to RVHT kidneys, STS significantly decreased TUNEL apoptosispositive stain cells in the cortex $(45.6 \%$ decrease in the RVHT STS group vs. the RVHT group) and medulla (50.6\% decrease in the RVHT STS group vs. the RVHT group) in the RVHT STS kidneys.

Sodium Thiosulfate and Renovascular Hypertension

\section{Discussion}

RVHT is commonly used as an experimental model for the study of hypertension. There are many different methods to induce hypertension with renal arterial constriction, for example, $1 \mathrm{~K} 1 \mathrm{C}, 2 \mathrm{~K} 1 \mathrm{C}$, and $2 \mathrm{~K} 2 \mathrm{C}$ methods [15]. This study was designed to utilize the $2 \mathrm{~K} 2 \mathrm{C}$ rat model developed as a model of RVHT through the activation of the RAAS system, sodium/volume overload, and sympathetic nervous system excitation [1-3]. A previous study has found that $1 \mathrm{~K} 1 \mathrm{C}$ RVHT had high mortality rate [18], whereas in the $2 \mathrm{~K} 1 \mathrm{C}$ RVHT model, the development of 
arterial blood pressure is relatively low and hard to achieve hypertension [19]. However, in the 2K2C RVHT model, there are 2 silver clips and 2 remaining kidneys in each rat, so the deficiency inherent in the 1-clip method can be avoided, and the expected high BP is easy to achieve in the $2 \mathrm{~K} 2 \mathrm{C}$ model with very few or no deaths as a result of acute renal failure [15]. The intention was to determine whether traditionally used STS might ameliorate RVHT-induced hypertension and renal oxidative damage by antihypertensive, antioxidant, and antiapoptotic action. Our data support the hypothesis that STS treatment could attenuate hypertension, renal oxidative stress, fibrosis, and apoptosis formation in our 2K2C RVHT model.

ROS are produced by the aerobic metabolism. The imbalance between production of ROS and antioxidant defense in any cell compartment is associated with cell damage and may play an important role in the pathogenesis of renal disease. NADPH oxidase family is the major ROS source in the vasculature and modulates renal perfusion. Elevation of angiotensin II and adenosine activates $\mathrm{NADPH}$ oxidase via angiotensin II type 1 receptors and adenosine adenosine 1 receptors in renal microvessels, leading to superoxide production [20]. Oxidative stress in the kidney prompts renal vascular remodeling and increases preglomerular resistance leading to hypertension and acute and chronic kidney injury. ROS increase in hypertension results in upregulation of renal afferent arteriole vasoconstriction, enhancement of myogenic responses, and change of tubuloglomerular feedback, which further promotes hypertension and nephropathy [20]. Yildirim et al. [21] implicated leukocyte-associated angiotensin type 1 receptor and NADPHoxidase gp91 (phox) in the induction of the pro-oxidative, proinflammatory, and prothrombogenic phenotype assumed by microvessels that are chronically exposed to elevated angiotensin II. Our data in the 2K2C RVHT model also demonstrated the significant elevation of NADPH oxidase gp91 and 4-HNE in the cortex and medulla in the RVHT kidneys implicating the increased oxidative stress in the damaged kidneys. Our results provided further important information that STS has direct scavenging ROS activity and suppressed NADPH oxiase gp91 activity and 4-HNE lipid peroxidation in both the cortex and medulla, as shown in Figure 1. Inhibition of NADPH oxidase reduces fibronectin expression in stroke-prone renovascular hypertensive rat brain in one previous report [22], implicating the possible role of STS on inhibition of fibrosis. Our data also informed that the antioxidant, antiapoptotic, and antifibrotic effects of STS seem to confer more locally renal protection in reducing proteinuria than in reducing sys- tematically arterial blood pressure levels. Because a less leukocyte infiltration, NADPH oxidase gp91 and 4-HNE expression, and fibrosis were demonstrated in the renal cortex and medulla with RVHT injury, we suggest that STS exerts more therapeutic potential on the decrease of kidney injury than that of hypertension. Therefore, the antioxidant STS may have an antioxidant effect on reduction in angiotensin II/angiotensin type 1 receptor/ NADPH oxidase gp91 (phox) signaling-mediated ROS production, vasoconstriction, inflammation, and fibrosis in the RVHT 2K2C model.

STS has been reported effective in treating calciphylaxis $[9,23]$, cyanide poisoning [10], cisplatin toxicity [11, 24], cerebral vasospasm [12], hypertension, and renal injury [13]. However, possible adverse effects of STS have been mentioned. Intravenous STS appeared to be the most common and acceptable mode of administration. However, patients with intravenous STS appeared to be more prone to sepsis (20\%) [25]. In addition, intravenous STS can lead to changes in osmotic pressure, followed by the release of $\mathrm{H}$ ions, resulting in acidosis [26]. Previous literature also suggested that intraperitoneal injection of STS was a cause of chemical peritonitis [25,27]. Several reports stated that STS via the pre-conditioning or postconditioning method was protective against rat heart ischemia/reperfusion-induced apoptosis and oxidative stress $[28,29]$. Shirozu et al. [30] indicated that administration of STS prevents acute inflammatory liver failure by augmenting thiosulfate levels and upregulating antioxidant and antiapoptotic defense in the liver. According to our survey, the exact role of STS on reduction of RVHTinduced renal apoptosis has not been clearly explored. In the present study, we found that STS can reduce RVHTinduced blood ROS amount, renal NADPH oxidase gp91, 4-HNE expression, Bax and PARP expression, and subsequent apoptosis production in the impaired kidneys, implicating STS reducing the proapoptosis-related mechanism by inhibiting the Bax/caspase 3/PARP signaling pathway. Based on the above information, STS has a wide range of protection against oxidative stress-evoked apoptosis induction in the heart, liver, and kidney.

A drug that is administered intravenously can be used only in several situations in human medicine. In an acute situation, STS does not decrease blood pressure enough in our results. Target organ damage needs more time to develop; therefore, STS cannot be used for prevention. In a chronic situation when no other options are available, it is vital to achieve improvement in the patients. This is also not true for high blood pressure as there are many drugs more suitable for treatment of hypertension and
Kidney Blood Press Res 2021;46:41-52 DOI: $10.1159 / 000510047$
Chou et al. 
target organ damage. The dosage, the administered route, or the duration of STS treatment should be determined in the future. However, STS may have a protective potential effect against oxidative stress-evoked oxidative injury via oral administration or other pathways, which should be explored in the future.

\section{Conclusion}

In summary, STS treatment can mildly counteract RVHT-induced hypertension and renal injury by its antioxidant, anti-inflammatory, antifibrotic, and antiapoptotic mechanisms. Treatment of STS may partly attenuate RVHT-induced oxidative stress via the depression of angiotensin II/angiotensin type 1 receptor/NADPH oxidase gp91/ROS signaling and inhibition of Bax/caspase 3/ PARP-mediated apoptosis in the kidneys.

\section{Statement of Ethics}

All surgical and experimental procedures were approved by the Institutional Animal Care and Use Committee of National Taiwan Normal University (Approval No. 107026, September 26, 2018) and were in accordance with the guidelines of the National Science Council of Republic of China.

\section{Conflict of Interest Statement}

The authors declare that they have no conflicts of interest.

\section{Funding Sources}

This work was partly supported by MOST-102-2320-B-003001-MY3 (Dr. Chiang-Ting Chien) and partly by a research fund of the Taipei Hospital (Dr. San-Chi Lin).

\section{Author Contributions}

P.-L.C., Y.-S.C., S.-C.L., and C.-T.C. fulfilled substantial contributions to conception and design. P.-L.C., Y.-S.C., S.-D.C., and C.-T.C. drafted and revised the article critically for important intellectual content. P.-L.C., S.-C.L., and C.-T.C. finally approved the manuscript to be published. P.-L.C. and Y.-S.C. contributed equally to the first author. S.-C.L. and C.-T.C. contributed equally to the corresponding author.

\section{Availability of Data and Material}

The analyzed data sets generated during the study are available from the corresponding author on reasonable request.

\section{References}

1 Mann SJ. Neurogenic hypertension: pathophysiology, diagnosis and management. Clin Auton Res. 2018;28(4):363-74.

2 Paravicini TM, Touyz RM. Redox signaling in hypertension. Cardiovasc Res. 2006;71(2): $247-58$.

3 Boshra V, Abbas AM. Effects of peripherally and centrally applied ghrelin on the oxidative stress induced by renin angiotensin system in a rat model of renovascular hypertension. J Basic Clin Physiol Pharmacol. 2017;28(4):347-54.

4 Sattar MA, Yusof AP, Gan EK, Sam TW, Johns EJ. Acute renal failure in $2 \mathrm{~K} 2 \mathrm{C}$ Goldblatt hypertensive rats during antihypertensive therapy: comparison of an angiotensin AT1 receptor antagonist and clonidine analogues. J Auton Pharmacol. 2000;20(5-6):297-304.

5 Zhu X, Zhou Z, Zhang Q, Cai W, Zhou Y, Sun $\mathrm{H}$, et al. Vaccarin administration ameliorates hypertension and cardiovascular remodeling in renovascular hypertensive rats. J Cell Biochem. 2018;119(1):926-37.

6 Muraya N, Kadowaki D, Miyamura S, Kitamura K, Uchimura K, Narita Y, et al. Benzbromarone attenuates oxidative stress in angiotensin II- and salt-induced hypertensive model rats. Oxid Med Cell Longev. 2018; 2018:7635274.
7 Chang HH, Hsu SP, Chien CT. Intrarenal transplantation of hypoxic preconditioned mesenchymal stem cells improves glomerulonephritis through anti-oxidation, anti-ER stress, anti-inflammation, anti-apoptosis, and anti-autophagy. Antioxidants. 2019;9(1):2.

8 Farese S, Stauffer E, Kalicki R, Hildebrandt T, Frey BM, Frey FJ, et al. Sodium thiosulfate pharmacokinetics in hemodialysis patients and healthy volunteers. Clin J Am Soc Nephrol. 2011;6(6):1447-55.

9 Hayden MR, Goldsmith DJ. Sodium thiosulfate: new hope for the treatment of calciphylaxis. Semin Dial. 2010;23(3):258-62.

10 Coentrao L, Moura D. Acute cyanide poisoning among jewelry and textile industry workers. Am J Emerg Med. 2011;29(1):78-81.

11 Sooriyaarachchi M, Narendran A, Gailer J. The effect of sodium thiosulfate on the metabolism of cis-platin in human plasma in vitro. Metallomics. 2012;4(9):960-7.

12 Thomas JE, McGinnis G. Safety of intraventricular sodium nitroprusside and thiosulfate for the treatment of cerebral vasospasm in the intensive care unit setting. Stroke. 2002;33(2): 486-92.
13 Snijder PM, Frenay AR, Koning AM, Bachtler M, Pasch A, Kwakernaak AJ, et al. Sodium thiosulfate attenuates angiotensin II-induced hypertension, proteinuria and renal damage. Nitric Oxide. 2014;42:87-98.

14 Bijarnia RK, Bachtler M, Chandak PG, van Goor H, Pasch A. Sodium thiosulfate ameliorates oxidative stress and preserves renal function in hyperoxaluric rats. PLoS One. 2015;10(4):e0124881.

15 Zeng J, Zhang Y, Mo J, Su Z, Huang R. Twokidney, two clip renovascular hypertensive rats can be used as stroke-prone rats. Stroke. 1998;29(8):1708-4.

16 Lee SP, Wu WY, Hsiao JK, Zhou JH, Chang $\mathrm{HH}$, Chien CT. Aromatherapy: activating olfactory calcium-sensing receptors impairs renal hemodynamics via sympathetic nervemediated vasoconstriction. Acta Physiol. 2019;225(1):e13157.

17 Hsu SP, Chen CC, Chien CT. Pretreatment of sialic acid efficiently prevents lipopolysaccharide-induced acute renal failure and suppresses TLR4/gp91-mediated apoptotic signaling. Kidney Blood Press Res. 2016;41(3): 267-77.
Sodium Thiosulfate and Renovascular Hypertension 
18 Ketabchi F, Bajoovand S, Adlband M, Naseh M, Nekooeian AA, Mashghoolozekr E. Right ventricular pressure elevated in one-kidney, one clip Goldblatt hypertensive rats. Clin Exp Hypertens. 2017;39(4):344-9.

19 Amiri F, Garcia R. Renal angiotensin II receptor regulation in two-kidney, one clip hypertensive rats: effect of ACE inhibition. Hypertension. 1997;30(3 Pt 1):337-44.

20 Xu N, Jiang S, Persson PB, Persson EA, Lai EY, Patzak A. Reactive oxygen species in renal vascular function. Acta Physiol. 2020 Apr 20; 229(4):e13477.

21 Yildirim A, Russell J, Yan LS, Senchenkova EY, Granger DN. Leukocyte-dependent responses of the microvasculature to chronic angiotensin II exposure. Hypertension. 2012; 60(6):1503-9.

22 Cui C, Chen AF, Jiang Z, Wu Q, Lin J, Wen $\mathrm{H}$, et al. Inhibition of $\mathrm{NAD}(\mathrm{P}) \mathrm{H}$ oxidase reduces fibronectin expression in stroke-prone renovascular hypertensive rat brain. Clin Exp Pharmacol Physiol. 2007;34(4):304-9.
23 Strazzula L, Nigwekar SU, Steele D, Tsiaras $\mathrm{W}$, Sise M, Bis S, et al. Intralesional sodium thiosulfate for the treatment of calciphylaxis. JAMA Dermatol. 2013;149(8):946-9.

24 Freyer DR, Frazier AL, Sung L. Sodium thiosulfate and cisplatin-induced hearing loss. N Engl J Med. 2018;379(12):1180-1.

25 Peng T, Zhuo L, Wang Y, Jun M, Li G, Wang $\mathrm{L}$, et al. Systematic review of sodium thiosulfate in treating calciphylaxis in chronic kidney disease patients. Nephrology. 2018;23(7): 669-75.

26 Selk N, Rodby RA. Unexpectedly severe metabolic acidosis associated with sodium thiosulfate therapy in a patient with calcific uremic arteriolopathy. Semin Dial. 2011;24(1): $85-8$
27 Gupta DR, Sangha H, Khanna R. Chemical peritonitis after intraperitoneal sodium thiosulfate. Perit Dial Int. 2012;32(2):220-2.

28 Ravindran S, Jahir Hussain S, Boovarahan SR, Kurian GA. Sodium thiosulfate post-conditioning protects rat hearts against ischemia reperfusion injury via reduction of apoptosis and oxidative stress. Chem Biol Interact. 2017;274:24-34.

29 Ravindran S, Boovarahan SR, Shanmugam K, Vedarathinam RC, Kurian GA. Sodium thiosulfate preconditioning ameliorates ischemia/reperfusion injury in rat hearts via reduction of oxidative stress and apoptosis. Cardiovasc Drugs Ther. 2017;31(5-6):51124.

30 Shirozu K, Tokuda K, Marutani E, Lefer D, Wang R, Ichinose F. Cystathionine $\gamma$-lyase deficiency protects mice from galactosamine/ lipopolysaccharide-induced acute liver failure. Antioxid Redox Signal. 2014;20(2):20416. 